\title{
A LOW-COST PAPER-BASED MODEL FOR ON-CHIP HUMAN RESPIRATORY SYSTEM STUDIES
}

\author{
Rahim Rahimi ${ }^{1}$, Manuel Ochoa ${ }^{1}$, Su Su Htwe ${ }^{2}$,Amy Donaldson ${ }^{2}$, Michael Zieger ${ }^{3}$, Mehmet R.Dokmeci ${ }^{4}$, \\ Ali Khademhosseini ${ }^{4}$, Rajiv Sood ${ }^{3}$, Amir Ghaemmaghami ${ }^{2}$, and Babak Ziaiel \\ ${ }^{1}$ Purdue University, West Lafayette, Indiana, USA \\ ${ }^{2}$ Queen's Medical Centre, University of Nottingham, Nottingham NG7 2UH, UK \\ ${ }^{3}$ Indiana University School of Medicine, Division of Plastic Surgery, IN, USA \\ ${ }^{4}$ Harvard-MIT Health Sciences and Technology, Cambridge, MA, USA
}

\begin{abstract}
We present the use of hydrophobic paper as an effective semipermeable membrane, ideal for air-liquid interface cell culture applications. The surface properties of the paper is modified through selective $\mathrm{CO}_{2}$ laser-assisted scrubbing to create a unique porous substrate with hydrophilic regions that regulates fluid diffusion and cell attachment. To select the appropriate model, four promising hydrophobic films were compared with each other in terms of gas permeability and long-term strength in an aqueous environment (wet-strength). Among the investigated substrates, parchment paper showed the fastest rate of oxygen diffusion. Mechanical analysis revealed superior dry and wet tensile strength of $69.5 \mathrm{MPa}$ and $29 \mathrm{MPa}$ for parchment paper which remained virtually unchanged after 7 days of submersion in PBS. The final platform with a constant flow of media in the basal lumen was found to show confluent coverage of Calu-3 (airway epithelial cells) with a mature $\mathrm{ZO} 1$ expression at the air-liquid interface after 7 days of cell culture.
\end{abstract}

\section{INTRODUCTION}

Respiratory diseases (e.g., pneumonia, tuberculosis, and lung cancer) are among the leading causes of mortality worldwide [1], [2]. The scarcity of current therapies calls for the development of more clinically relevant models to facilitate the study of the respiratory system and drug testing. To aid such understanding and to develop appropriate therapeutics, researchers are limited to animal and transwell-based in-vitro models for epithelial tissue. The restricted control of experimental variables and biological irrelevancy of animal models can result in both time consuming and unreliable data. Therefore, in-vitro models that mimic the functionality and physiological properties of the respiratory epithelia can provide a better understanding of the drug efficacy at the cellular level [3]. Currently, the most common in-vitro approach is the use of commercially available semipermeable hanging film for creating an air-liquid interface (ALI) in transwell flasks [4]. This technique has proven to be physiologically invaluable for the development of epithelial therapeutics; however, it does not constitute an ideal (and practical) epithelial model due to its inability to faithfully recapitulate the mechanics of epithelial tissue (e.g., most are brittle and too impermeable to gases, or not sufficiently strong).

Recently, many researchers have attempted to fabricate microengineered models that mimics the structure and functionality of the various physiological environments by using micro-fabrication technology. These efforts have resulted in the development of several examples of organ-on-a-chip technologies including lung, brain, kidney, and cancer [5]-[7]. However, many of these require time consuming fabrication processes and highcost cleanroom manufacturing, limiting the translation of the technology to high-throughput pharmaceutical research industry. A more practical approach (clinically and financially) would be one which takes advantage of modern rapid prototyping equipment for low-cost production and customization as well as suitable commercially available materials which are inexpensive, ubiquitous, and biocompatible [8].

As a more cost effective approach, many researcher have successfully shown the use of paper as an alternative material for conventional cell culture substrates (e.g., polystyrene, and PDMS) [9], due to its naturally biocompatible $3 \mathrm{D}$ cellulose fiber composition and its webbed architecture for efficient cell attachment [10], [11]. However, the inherent hydrophilic nature and low mechanical strength when wet, prevents it's prolong use in aqueous environments [12]. Nevertheless, impregnating paper with hydrophobic materials (e.g., wax, silicone) imparts on it unique mechanical and fluidic properties which mimic those of epithelial tissue and which can be conveniently integrated with BioMEMS devices [13]. In this study, we expand the benefit of paper-based biological assays by presenting a model for epithelial tissue consisting of a commercial hydrophobic paper (parchment paper) which is further customized via laser machining to create a unique substrate for epithelial cell culture and attachment, Figure 1a.

A schematic of the working mechanism of the microfluidic airway systems is demonstrated in Figure 1b. The system consist of an upper and a lower laser cut acrylic chambers corresponding

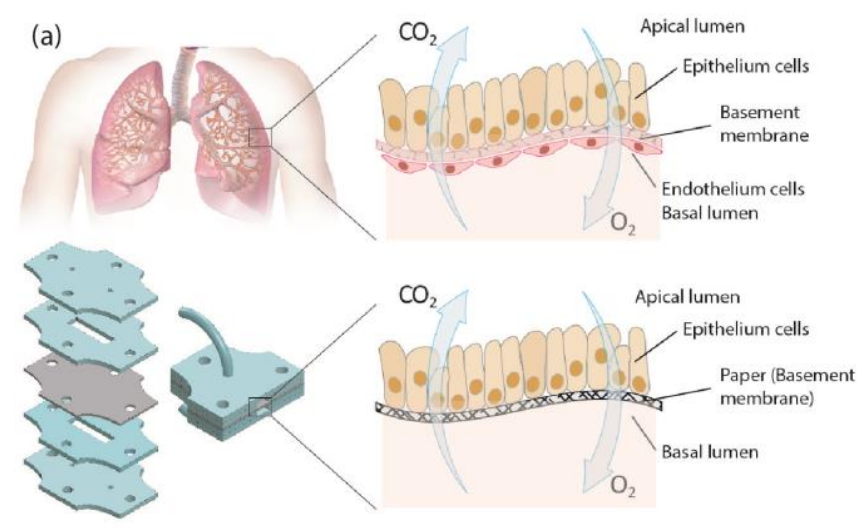

(b)

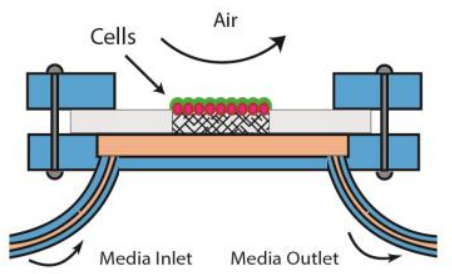

Figure 1: (a) Illustration of the respiratory epithelial tissue and paper-based microfabricated lung-mimicking device, (b) schematic of the air-liquid-interface (ALI) platform. 
to the apical (airway lumen) and basal compartments of airway epithelium. The laser-treated hydrophobic paper provides a semipermeable support for the cell attachment with regulated permeability of both oxygen and nutrition, mimicing the in-vivo basement membrane.

\section{EXPERIMENTAL}

To compare different substrates for the described airway model, we characterized four different promising common films in terms of their wet-strength (standard tensile strain as a function of wetting duration) and gas permeability. These films were wax paper, copy paper, parchment paper, PDMS, and a paper-PDMS composite prepared by placing a sheet of filter paper on a thin layer of $50 \mu \mathrm{m}$ spin-coated PDMS pre-polymer. Standard tensile stress-strain test was used to characterize how well the fibers in different papers hold together after the wetted for various number of days. In this test, the papers were submerged in buffer saline solution (PBS) for various durations (0-7days) and the ultimate tensile strength (UTS) and Young's modulus were subsequently measured using a universal testing machine $\left(\right.$ Admet $^{\mathbb{R}}$, model eXpert 1000). All specimens were laser cut to the same $(5 \mathrm{~mm} \times$ $20 \mathrm{~mm}$ ) dimensions. The tensile strength measurements were performed by fixing the two ends of the film and stretching from $0 \%$ to $12 \%$ strain at a constant extension velocity of $10 \mathrm{~mm} / \mathrm{min}$.

A customized setup was used to measure the gas permeability through different films, Figure 2. The structure consisted of a cylindrical chamber filled with $20 \mathrm{ml}$ deoxygenated DI water and covered by $20 \mathrm{~mm}$ in diameter circular sample of the film. For all tests, the dissolved oxygen was removed by purging the water for $8 \mathrm{~h}$ with nitrogen. The oxygen permeability was confirmed with real time measurement of the dissolved oxygen using an optical oxygen sensor positioned in the DI water chamber. All measurements were performed at room temperature and atmospheric pressure. We evaluated the oxygen permeability of different membrane via the rate of dissolve oxygen increase in the water. Figure 3 shows the optical photographs of the paper-based microfluidic airway systems. In this platform, the laser patterned hydrophobic paper with hydrophilic traces were placed between to laser-cut acrylic chambers $(1.5 \mathrm{~mm}$ deep and $15 \mathrm{~mm}$ long channels). As the first step, each component of ALI platform including parchment paper were sterilized by irradiating with ultraviolet (UV) light at a distance of $8 \mathrm{~cm}$ for $15 \mathrm{~min}$. The ALI platform was assembled as shown in the Figure 3 inset. The inletoutlet tubing were connected to platform after sterilization with $70 \%$ ethanol. An antibiotic/antimycotic solution was placed in the upper/ lower chambers and interface tubing overnight at room temperature inside the tissue culture hood. The sterilizing solution

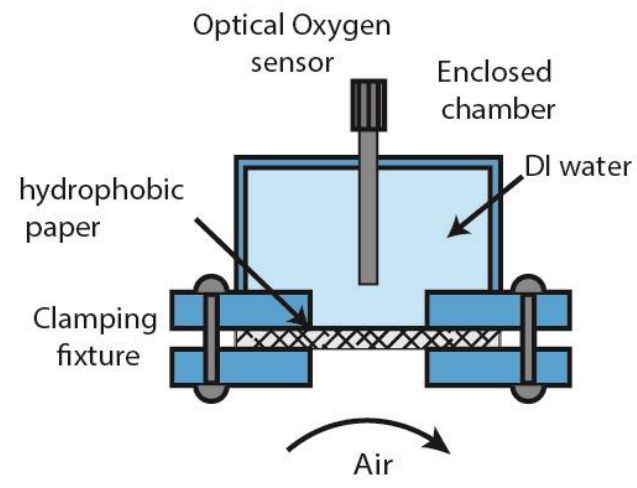

Figure 2: Gas permeability test setup. was removed and the chambers and tubing were washed with PBS twice. Next the top open chamber was filled with media and seeded at a density of $1.5 \times 10^{6}$ cells $/ \mathrm{ml}$ in $200 \mu$ with Airway Epithelial Cells (Calu-3) and the bottom chamber was infused with a constant supply of media at a flow rate of $8 \mu \mathrm{l} / \mathrm{min}$. The whole assembly was incubated at $37{ }^{\circ} \mathrm{C}, 5 \% \mathrm{CO}_{2}$ under static condition. Once the cells exhibited a confluent monolayer coverage on the hydrophilic regions of the paper $(5 \mathrm{~mm} \times 15 \mathrm{~mm})$ an air-liquid interface was established by removing media from the top chamber while maintain a constant flow of media in the bottom chamber for 7 days. Live-dead assay was performed with the biohazard cell viability kit (Life technologies) which contains SYTO 10 (green fluorescent nucleic acid stain for live cells) and DEAD Red (ethidium homodimer-2, nucleic acid stain for dead cells). The dye mixture was prepared in 1:500 dilution of SYTO 10 and 1: 1000 dilution of DEAD Red. Before addition of dye mixture, all samples were washed with PBS for 1 time. In order to assess the airway epithelium's differentiation that closely resembles native airway tissue, the cells were stained for ZO1 (bright green), pancytokeratin marker (panKRT-green) and DAPI (nuclear staining blue) at 5days and 7days in ALI under static and flow conditions. The static condition was used as a model for studying lung injuries induced by thromboembolism (pulmonary embolism), which the pulmonary arteries are blocked and blood flow is in a static condition.

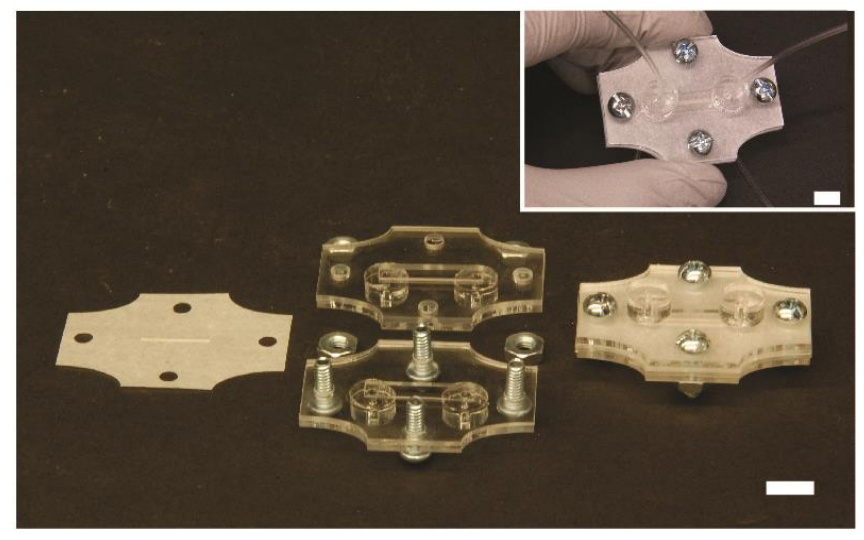

Figure 3: Photograph of components and final assembled onchip microfluidic respiratory epithelia tissue model (inset). All scale bars: $10 \mathrm{~mm}$.

\section{RESULTS AND DISCUSSION \\ Mechanical characterization}

Figure 4 shows the stress-strain curve for filter paper and various hydrophobic papers before and after $24 \mathrm{~h}$ submerging in PBS. All the samples in dry states show a linear stress-strain profile with a small strain $(2.5 \%)$ before rupture. The sharp drop in the plot specifies the strain level at which the paper splits in half. The commercial parchment paper and wax paper have the highest mechanical properties in dry state with the UTS of $69.4 \mathrm{MPa}$ and $73 \mathrm{MPa}$ and Young's moduli of $33.2 \mathrm{GPa}$ and $33.4 \mathrm{GPa}$, respectively. Filter paper impregnated with PDMS has a dry UTS of $21.4 \mathrm{MPa}$ which is three time higher than the pristine filter paper (7.6 MPa). This increase in mechanical strength is explained by the presence of the PDMS filler in the network fiber of the paper forming a stronger composite film. Wet tensile strength results, for all the specimens, show an increase in elasticity but decrease in mechanical strength. 
Unlike the dry state, the wet papers show and an average $6 \%$ strain followed by necking before rupture. This is due to the diffusion and plasticizing effect of water molecules in the paper film. The results show that the hydrophobic papers retain some of their mechanical strength after $24 \mathrm{~h}$ submerging in PBS, whereas the filter paper wet UTS drastically decreases to $1.2 \mathrm{MPa}$ and starts to disintegrate in the solution. Among the investigated hydrophobic papers, parchment paper retains more than $40 \%$ of its original dry UTS strength followed by PDMS/paper and wax paper with retentions of $31 \%$ and $14 \%$, respectively. The trend in change in UTS for different films as a function of wetting duration is shown in Figure 5. The parchment paper and wax paper show a stable retention of mechanical strength (UTS) of $29 \mathrm{MPa}$ and 8.9 MPa for 7 days. However, for filter paper and PDMS/paper, a longer wetting duration reduces the mechanical strength (UTS) down to $0.4 \mathrm{MPa}$ and $5.4 \mathrm{MPa}$, respectively.
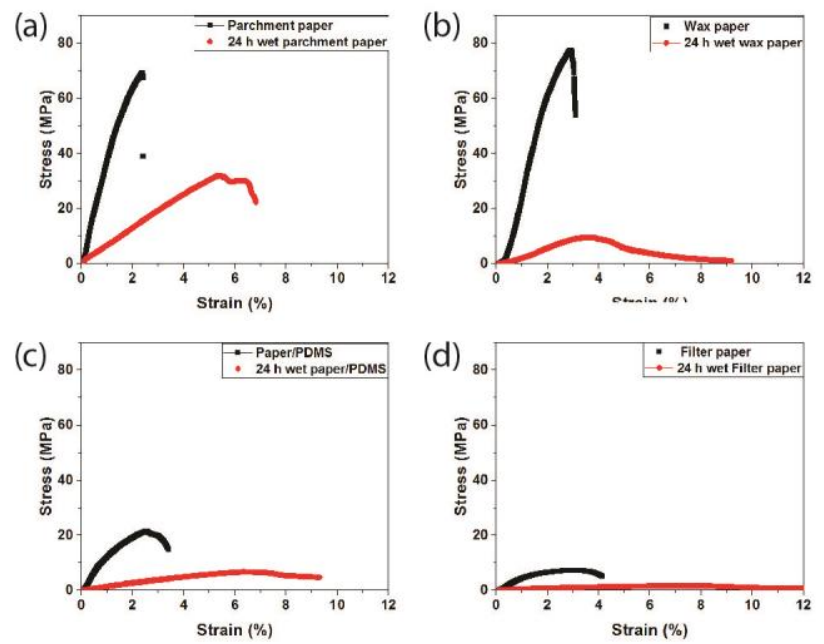

Figure 4: Stress vs. strain of dry and wet paper films after 24 hours of PBS immersion (a) parchment paper, (b) wax paper, (c) paper-PDMS, and (d) filter paper.

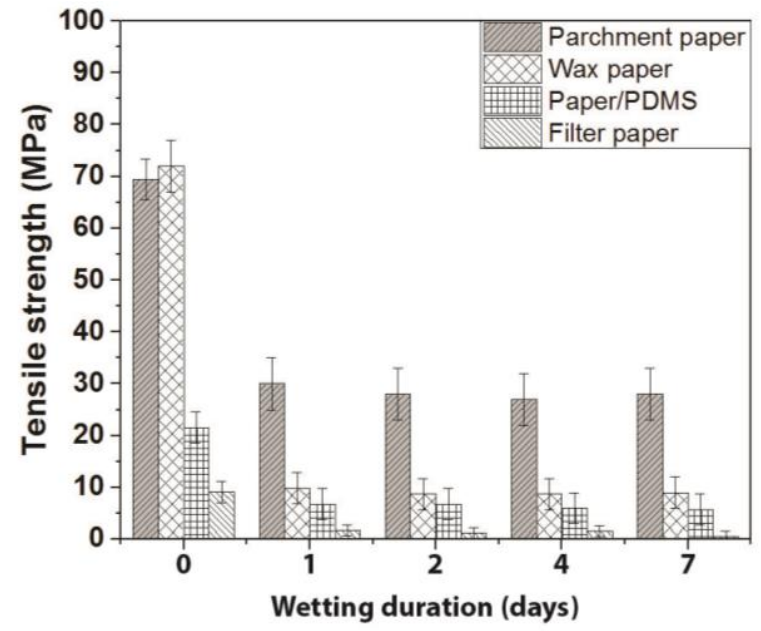

Figure 5: Ultimate tensile strength for different papers as a function of wetting duration.

\section{Oxygen permeability characterization}

Figure 6 shows the gas permeability results for different films using the aforementioned setup (Figure 2). For all measurements the initial dissolved oxygen of the DI water was close to zero $(\sim 0.5 \mathrm{ppm})$ and increased with time up to the oxygen saturation level in the water ( $8 \mathrm{ppm})$. The increase is due to the diffusion of the oxygen gas in ambient condition through the membrane and its dissolution in the water. Without any membrane, the water equilibrates to its steady-state saturation level of about $8 \mathrm{ppm}$ in less than $140 \mathrm{~min}$. However, when the chamber is covered, the time required for oxygen saturation increases. The results show the longest oxygen equilibration time occurs for a pristine $100 \mu \mathrm{m}$ membrane of PDMS (720 min), and the shortest time occurs for parchment paper $(210 \mathrm{~min}$, with an average rate of $2.4 \mathrm{ppm} / \mathrm{h})$. No signs of water leakage were observed with the hydrophobic films during any of the measurements. The mechanical strength and gas permeability results show the superior performance of the parchment paper, providing a suitable substrate for the proposed ALI platform.

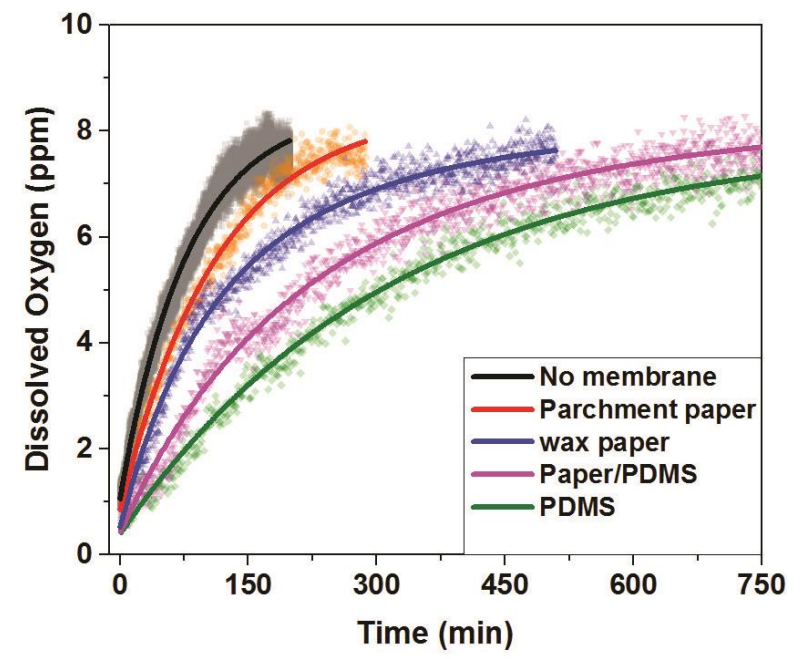

Figure 6: Oxygen permeability of different hydrophobic paper membranes.

\section{Cell culture results}

As shown in Figure 7a, with the aid of live (green) and dead (red) staining the CALU3 cells exhibited a confluent monolayer coverage with high cell viability $(>90 \%)$ after 3 days. In continuous flow condition the mature ZO1 expression (chicken wire appearance) was more prominent after 7 days culture compared to the 5 day ALI samples, Figure $7 \mathrm{~b}$. ZO1 is the tight junction protein that is mainly expressed in the differentiated airway epithelium. The results show a much faster epithelium differentiation compared to commercial transwell plates which often require 30 days for complete differentiation. However, in static ALI condition, there is no sign of ZO1 staining, and most of the cells die rather than fully differentiating, Figure $7 \mathrm{c}$. The results show that parchment paper provides an ideal, robust, and inexpensive platform for generating epithelial cells on-chip in a high-throughput fashion for disease modelling and in-vitro drug testing. 

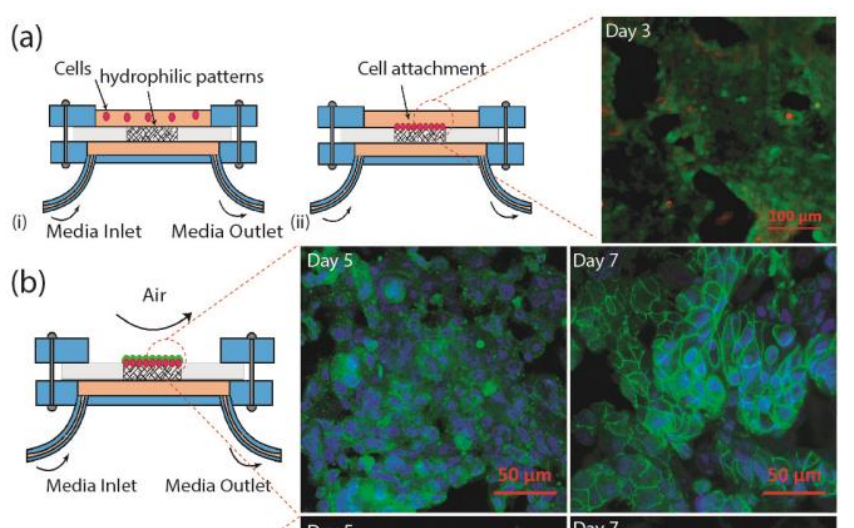

(c)
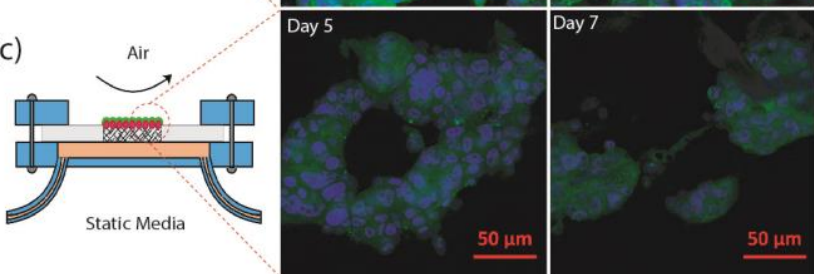

Figure 7: (a) Assessment of cell attachment and viability after 3 days, (b) ZO1 expression (bright green) on differentiated CALU3 cells at Air Liquid Interface for 5 and 7days under flow condition and (c) under static condition.

\section{CONCLUSIONS}

In this study, we investigated the potential use of a paperbased microfluidic system as an in-vitro model for human respiratory system studies. Among different papers, parchment paper showed superior performance in terms of wet strength and gas permeability. Furthermore, by selectively treating the surface of this hydrophobic paper with a $\mathrm{CO}_{2}$ laser, we were able to produce hydrophilic regions with effective cell attachment and to regulate fluid diffusion which mimics the semi-permeable properties observed in basement membrane of human respiratory system. The final device, composed of parchment paper and acrylic microfluidics, was able to maintain long-term stability under constant flow of media with faster differentiation rate of airway epithelium compared to conventional transwell methods. This cost-effective platform can facilitate new drug discovery and improve the understanding of the etiology for different diseases by providing a model that better mimics the respiratory surface at the cellular level.

\section{ACKNOWLEDGEMENTS}

Funding for this work was provided by the National Science Foundation under grant EFRI-BioFlex \#1240443.

\section{REFERENCES}

[1] C. A. Pope, "Cardiovascular Mortality and Long-Term Exposure to Particulate Air Pollution: Epidemiological Evidence of General Pathophysiological Pathways of Disease," Circulation, vol. 109, no. 1, pp. 71-77, Dec. 2003.

[2] W. W. Thompson, "Mortality Associated With Influenza and Respiratory Syncytial Virus in the United States," JAMA, vol. 289, no. 2, p. 179, Jan. 2003.

[3] H. Harrington, P. Cato, F. Salazar, M. Wilkinson, A. Knox, J. W. Haycock, F. Rose, J. W. Aylott, and A. M.
Ghaemmaghami, "Immunocompetent 3D Model of Human Upper Airway for Disease Modeling and In Vitro Drug Evaluation.," Mol. Pharm., vol. 11, no. 7, pp. 2082-91, Jul. 2014.

[4] Y. Zhu, A. Chidekel, and T. H. Shaffer, "Cultured Human Airway Epithelial Cells (Calu-3): A Model of Human Respiratory Function, Structure, and Inflammatory Responses," Crit. Care Res. Pract., vol. 2010, pp. 1-8, 2010.

[5] D. Huh, H. Fujioka, Y. Tung, N. Futai, R. Paine, J. B. Grotberg, and S. Takayama, "Acoustically detectable cellular-level lung injury induced by fluid mechanical stresses in microfluidic airway systems.," Proc. Natl. Acad. Sci. U. S. A., vol. 104, no. 48, pp. 18886-91, Nov. 2007.

[6] S. N. Bhatia and D. E. Ingber, "Microfluidic organs-onchips.," Nat. Biotechnol., vol. 32, no. 8, pp. 760-772, Aug. 2014.

[7] D. D. Nalayanda, C. Puleo, W. B. Fulton, L. M. Sharpe, T.H. Wang, and F. Abdullah, "An open-access microfluidic model for lung-specific functional studies at an air-liquid interface.," Biomed. Microdevices, vol. 11, no. 5, pp. 10819, Oct. 2009.

[8] G. E. Morris, J. C. Bridge, L. A. Brace, A. J. Knox, J. W. Aylott, C. E. Brightling, a M. Ghaemmaghami, and F. R. a J. Rose, "A novel electrospun biphasic scaffold provides optimal three-dimensional topography for in vitro co-culture of airway epithelial and fibroblast cells.," Biofabrication, vol. 6, no. 3, p. 035014, 2014.

[9] K. A. Simon, K. M. Park, B. Mosadegh, A. B. Subramaniam, A. D. Mazzeo, P. M. Ngo, and G. M. Whitesides, "Polymer-based mesh as supports for multilayered 3D cell culture and assays," Biomaterials, vol. 35, no. 1, pp. 259-268, Jan. 2014.

[10] R. Derda, A. Laromaine, A. Mammoto, S. K. Y. Tang, T. Mammoto, D. E. Ingber, and G. M. Whitesides, "Papersupported 3D cell culture for tissue-based bioassays.," Proc. Natl. Acad. Sci. U. S. A., vol. 106, no. 44, pp. 18457-62, Nov. 2009.

[11] S. Ahmed, M. N. Bui, and A. Abbas, "Paper-based chemical and biological sensors: Engineering aspects," Biosens. Bioelectron., vol. 77, pp. 249-263, Mar. 2016.

[12] H. Juvonen, A. Määttänen, P. Laurén, P. Ihalainen, A. Urtti, M. Yliperttula, and J. Peltonen, "Biocompatibility of printed paper-based arrays for 2-D cell cultures," Acta Biomater., vol. 9, no. 5, pp. 6704-6710, May 2013.

[13] B. Mosadegh, M. R. Lockett, K. Thu, K. A. Simon, K. Gilbert, S. Hillier, D. Newsome, H. Li, A. B. Hall, D. M. Boucher, B. K. Eustace, and G. M. Whitesides, "Biomaterials A paper-based invasion assay: Assessing chemotaxis of cancer cells in gradients of oxygen," Biomaterials, vol. 52, pp. 262-271, 2015.

\section{CONTACT}

*B.Ziaie, tel:+1-765-404-0726; bziaie@purdue.edu 